\title{
Clinical utility gene card for: Long-QT syndrome (types 1-13)
}

\author{
Britt-Maria Beckmann ${ }^{\star, 1,4}$, Arthur AM Wilde ${ }^{2}$ and Stefan Kääb ${ }^{\star, 1,3,4}$ \\ European Journal of Human Genetics (2013) 21, doi:10.1038/ejhg.2013.28; published online 20 March 2013
}

\section{DISEASE CHARACTERISTICS}

1.1 Name of the disease (synonyms)

Long-QT syndrome (LQT, LQTS, Romano-Ward syndrome, subgroups: Jervell and Lange-Nielsen syndrome, Andersen syndrome, Timothy syndrome).

\subsection{OMIM\# of the disease}

192500 (LQT1); 613688 (LQT2); 603830 (LQT3); 600919 (LQT4); 613695 (LQT5); 613693 (LQT6); 170390 (LQT7), Andersen syndrome; 601005 (LQT8), Timothy syndrome; 611818 (LQT9); 611819 (LQT10); 611820 (LQT11); 612955 (LQT12); 613485 (LQT13); 220400 Jervell and Lange-Nielsen syndrome 1, JLNS1; 612347 Jervell and Lange-Nielsen syndrome 2, JLNS2

1.3 Name of the analysed genes or DNA/chromosome segments LQT1: KCNQ1, 11p15.5; LQT2: KCNH2, 7q35-q36; LQT3: SCN5A, 3p21; LQT4: ANK2, 4q25-q27; LQT5: KCNE1, 21q22.1-q22.2; LQT6: KCNE2, 21q22.1; LQT7: KCNJ2, 17q23.1-q24.2; LQT8: CACNA1C, 12p13.3; LQT9: $C A V 3,3$ p25; LQT10: $S C N 4 B, 11 \mathrm{q} 23$; LQT11: AKAP9, 7q21-q22; LQT12: SNTA1, 20q11.2; LQT13: KCNJ5, 11q24; JLNS1: KCNQ1, homozygous or compound heterozygous, 11p15.5; JLNS2: KCNE1, homozygous or compound heterozygous, 21q22.

\subsection{OMIM\# of the gene(s)}

607542 (LQT1), 152427 (LQT2), 600163 (LQT3), 106410 (LQT4), 176261 (LQT5), 603796 (LQT6), 600681 (LQT7), 114205 (LQT8), 601253 (LQT9), 608256 (LQT10), 604001 (LQT11), 601017 (LQT12), 600734 (LQT13).

\subsection{Mutational spectrum}

The mutation detection rate is about $70 \%$. If a mutation is found the spectrum is as follows: $42-52 \%$ mutations in KCNQ1 (16 exons); 32-45\% mutations in KCNH2 (15 exons); 8-13\% mutations in SCN5A (28 exons); rare: mutations in ANK2 (46 exons); rare: mutations in KCNE1 (1 exon); rare: mutations in KCNE2 (1 exon); rare: mutations in KCNJ2 (2 exons); rare: mutations in CACNA1C; rare: mutations in CAV3 (2 exons); rare: mutations in SCN4B (5 exons); rare: mutations in AKAP9 (51 exons); rare: mutations in SNTA1 (8 exons); rare: mutations in KCNJ5 (3 exons).

The mutational spectrum contains practically all types of mutations (missense, frameshift, nonsense, splice site, deletions and insertions).
Most patients are heterozygous for a mutation, but in $\sim 5 \%$ of the cases, patients carry two mutations in the same (compound heterozygous or homozygous) or in different genes (digenic). The percentage of involved genes is slightly different from country to country. In the Netherlands, for example, LQT2 is more prevalent $(>50 \%)$. $^{1-3}$

\subsection{Analytical methods}

Direct sequencing, in addition MLPA for detection of larger deletions or duplications.

It is foreseeable that NGS technologies will increasingly be used. However, there is still variability concerning sensitivity and specificity within NGS platforms and different software. Besides, the detection of insertions and deletions has proven to be more difficult. Therefore, most laboratories still do not offer this type of analysis for diagnostic purposes in the clinical setting.

\subsection{Analytical validation}

Sequencing of both strands and independent analysis of a freshly prepared second patient sample.

\subsection{Estimated frequency of the disease}

(Incidence at birth ('birth prevalence') or population prevalence. If known to be variable between ethnic groups, please report): 1:2,000 individuals in the general population. ${ }^{4}$ It may be assumed that the prevalence is of comparable magnitude in different populations.

\subsection{Diagnostic setting:}

\begin{tabular}{lll}
\hline & Yes & No \\
A. (Differential) diagnostics & $\bigotimes$ & $\square$ \\
B. Predictive testing & $\bigotimes$ & $\square$ \\
C. Risk assessment in Relatives & $\bigotimes$ & $\square$ \\
D. Prenatal & $\bigotimes$ & $\square$
\end{tabular}

\section{Comment:}

Prenatal diagnosis of Long-QT syndrome is indicated in very exceptional situations only and is asked for extremely rarely.

\footnotetext{
${ }^{1}$ Department of Medicine 1, University Hospital of the Ludwig Maximilians University-Campus Grosshadern, Munich, Germany; ${ }^{2}$ Department of cardiology AMC, Amsterdam, The Netherlands; ${ }^{3}$ Munich Heart Alliance, Munich, Germany

${ }^{4}$ These authors contributed equally to this work.

*Correspondence: Dr BM Beckmann, Department of Medicine 1, University Hospital of the Ludwig Maximilians University-Campus Grosshadern, Marchioninistrasse 15, Munich 81377, Germany. Tel: +498970952251; Fax: +498970955251; E-mail: brittmaria.beckmann@med.uni-muenchen.de or Dr S Kääb, Department of Medicine 1, University Hospital of the Ludwig Maximilians University-Campus Grosshadern, Marchioninistrasse 15, Munich 81377 , Germany. Tel.: +49 89 7095 3060 ; E-mail: stefan.kaab@med.uni-muenchen.de
} 


\section{TEST CHARACTERISTICS}

\begin{tabular}{|c|c|c|c|c|}
\hline & \multicolumn{2}{|c|}{ Genotype or disease } & \multirow{2}{*}{$\begin{array}{l}\text { A: True positives } \\
\text { B: False positives }\end{array}$} & \multirow{2}{*}{$\begin{array}{l}\text { C: False negatives } \\
\text { D: True negatives }\end{array}$} \\
\hline & Present & Absent & & \\
\hline \multicolumn{5}{|l|}{ Test } \\
\hline \multirow[t]{2}{*}{ Positive } & $A$ & B & Sensitivity: & $A /(A+C)$ \\
\hline & & & Specificity: & $D /(D+B)$ \\
\hline \multirow[t]{2}{*}{ Negative } & $\mathrm{C}$ & $\mathrm{D}$ & Positive predictive value: & $A /(A+B)$ \\
\hline & & & Negative predictive value: & $D /(C+D)$ \\
\hline
\end{tabular}

\subsection{Analytical sensitivity}

(proportion of positive tests if the genotype is present)

Close to $100 \%$ if complete Sanger sequencing and MLPA of the affected gene is performed.

But this nearly $100 \%$ analytical sensitivity includes causal mutations as well as variants that are just innocent bystanders where the clinical impact has to be proven subsequently. ${ }^{5}$

\subsection{Analytical specificity}

(proportion of negative tests if the genotype is not present)

Close to $100 \%$ if complete sequencing and MLPA of the affected gene is performed. But not finding a mutation rejects by no means the diagnosis LQTS in definite clinical cases as in about 30\% the causative genes are still not known.

\subsection{Clinical sensitivity}

\section{(proportion of positive tests if the disease is present)}

The clinical sensitivity can be dependent on variable factors such as age or family history. In such cases a general statement should be given, even if quantification can only be made case.

On average the mutation detection rate for the most frequent LQTS disease genes (KCNQ1, KCNH2, SCN5A, KCNE1 and KCNE2) is about $70 \%{ }^{6}$

\subsection{Clinical specificity}

(proportion of negative tests if the disease is not present)

About 95\%, however, the rate of rare variants of uncertain significance (i.e, non-synonymous genetic variation) is about $4 \%$ in caucasian and $8 \%$ in non-caucasian in the LQTS $1-3$ genes. $^{5}$

\subsection{Positive clinical predictive value}

(life-time risk to develop the disease if the test is positive)

Nearly $50-60 \%$, this seems to depend on genotype. Before the age of 40 , roughly $40 \%$ of patients with LQT1 and LQT 2 become symptomatic. In LQT3 this is less, but symptoms may be more severe. Phenotypic expression of the disorder is time-dependent and LQTS subjects maintain a high risk for life-threatening cardiac events after age 40 years, which seems to be less high for LQTS1. ${ }^{7,8}$

\subsection{Negative clinical predictive value} (probability not to develop the disease if the test is negative) Assume an increased risk based on family history for a non-affected person. Allelic and locus heterogeneity may need to be considered. Index case in that family had been tested:
If the index case in that family had been tested and a causative mutation had been found in the index patient: close to $100 \%$.

Index case in that family had not been tested:

If the patient is clinically affected (prolonged QTc with or without syncope) he/she has a chance of about $70 \%$ carrying a disease-causing mutation. But only in very rare cases there is an indication for performing LQTS genetic testing in a clinically unaffected relative when the index case has not been tested.

This could be imaginable when in an index case there's a strong clinical suspicion of LQTS and there's no DNA available or the index patient refuses genetic testing. Usually there's no indication for genetic testing in a clinically unaffected family member with unclear genetic status of the index patient.

\section{CLINICAL UTILITY}

3.1 (Differential) diagnostics: The tested person is clinically affected (To be answered if in 1.9 ' $A$ ' was marked)

\subsubsection{Can a diagnosis be made other than through a genetic} test?

\begin{tabular}{lll}
\hline No & $\square$ (continue with 3.1.4) & \\
Yes $\quad$ Q & \\
& Clinically & $\square$ \\
& Imaging & $\square$ \\
Endoscopy & $\square$ \\
& Biochemistry & $\square$ \\
Electrophysiology & $\square$ \\
& Other (please describe) & ECG recordings and typical clinical symptoms \\
& & (with low sensitivity and specificity)!9,10 \\
\hline
\end{tabular}

\subsubsection{Describe the burden of alternative diagnostic methods to the patient}

ECGs are a non-invasive procedure with no risks and little inconvenience for the patient. But for the reason of low sensitivity and specificity the burden is psychological: uncertainty of proper diagnosis as well as clinical: individual therapy, inividual recommendations for treatment, lifestyle adaption and individual risk stratification based on specific subtype are not possible.

\subsubsection{How is the cost effectiveness of alternative diagnostic methods to be judged?}

As far as a disease-causing mutation is identified in the index patient, genetic testing can be offered to apparently healthy relatives within the family in order to determine if they carry the same mutation and are at-risk for malignant ventricular arrhythmias. If the relative carries the mutation a prophylactic inexpensive medical treatment can be started and specific advice can be given to gene carriers (avoiding substances/drugs, which might trigger arrhythmias, avoidance of genotype-specific triggers for arrhythmias, careful attendance in case of pregnancy and delivery, reproductive counselling, counselling concerning choice of profession). There's a reduction of the relative risk for developing serious cardiac events of about $65 \%$ by proper treatment (mostly with an unexpensive betablocker therapy) and the cardiac events in untreated patients on the other hands may lead to early invalidity or death in otherwise healthy young people with high national economic loss.

Therefore, cost effectiveness for alternative methods with their low sensitivity and specificity is unjustifiably low. 
3.1.4 Will disease management be influenced by the result of a genetic test?

No $\square$

Yes $\otimes$

Therapy

(please

describe)

Pharmaceutical treatment (usually betablockers) as primary and secondary prevention. In rare individual cases additional pacemaker and/or an implantable cardioverter defibrillator, and/or left cardiac sympathetic denervation is used. The implantation of an implantable cardioverter defibrillator with or without the performance of left cardiac sympathetic denervation is mostly reserved for patients in which optimal noninvasive therapy and lifestyle modifications fail to protect against ventricular arrhythmias. Only in exceptional individual cases implantable cardioverter defibrillator implantation might be indicated for primary prevention.

Pharmacotherapy might differ between genotypes. Also, lifestyle advice is different for the different genotypes. ${ }^{11,12}$

Prognosis Regular preventive medical checkups to recognize disease (please progression earlier, and appropriate treatment improves progdescribe) nosis. On appropriate treatment and with appropriate lifestyle adjustments prognosis is good and may not differ from normal.

Management Regular preventive medical checkups of mutation carriers,

(please intensification of therapy and/or defibrillator implantation and/ describe) or left cardiac sympathetic denervation, if necessary.

The molecular-genetic information is very important for clinical management, especially for LQTS3 patients.

3.2 Predictive setting: The tested person is clinically unaffected but carries an increased risk based on family history (To be answered if in 1.9 ' $\mathrm{B}$ ' was marked)

3.2.1 Will the result of a genetic test influence lifestyle and prevention? If the test result is positive (please describe)

Regular cardiological checkups.

Lifestyle adjustment, avoiding event related triggers (for example, swimming in LQT1 and loud acoustic stimuli in LQT2). ${ }^{112}$ ) Avoiding stringent competition sports. Avoiding QT prolonging drugs (www.qtdrugs.org) and avoidance of fever.

Some mutations have an unusually high clinical severity (for example, KCNQ1 A341V). ${ }^{13}$ Also patients with compound heterozygous mutations and JLNS patients are at a higher risk.

If the test result is negative (please describe).

Precautionary measures as described above are not needed.

3.2.2 Which options in view of lifestyle and prevention does a person at-risk have if no genetic test has been done (please describe)?

Same as described above. However, the preventive measures are much better accepted and compliance is improved if a positive test result was obtained.

3.3 Genetic risk assessment in family members of a diseased person (To be answered if in 1.9 ' $\mathrm{C}$ ' was marked)

3.3.1 Does the result of a genetic test resolve the genetic situation in that family?

If a disease-causing mutation is found: yes. Otherwise, potentially affected relatives should undergo regular cardiological evaluation.

3.3.2 Can a genetic test in the index patient save genetic or other tests in family members?

No. But in case of a positive test result in the index patient, clinically asymptomatic relatives being non-carriers of the pathogenic mutation can be excluded from regular cardiological follow-up.

3.3.3 Does a positive genetic test result in the index patient enable a predictive test in a family member?

Yes.

\subsection{Prenatal diagnosis}

(To be answered if in 1.9 ' $\mathrm{D}$ ' was marked)

\subsubsection{Does a positive genetic test result in the index patient enable a} prenatal diagnosis?

Yes, but prenatal diagnostics are not actively offered.

\section{IF APPLICABLE, FURTHER CONSEQUENCES OF TESTING}

Please assume that the result of a genetic test has no immediate medical consequences. Is there any evidence that a genetic test is nevertheless useful for the patient or his/her relatives? (Please describe).

For every patient (clinically affected or not) there are known specific triggers for arrhythmias to be avoided (e.g., QT prolonging drugs, competitive sports, low potassium serum levels, swimming in LQT1, sudden loud noise in LQT2). ${ }^{11,12}$ Thus, there should be thorough counselling concerning lifestyle modifications and choice of employment.

\section{CONFLICT OF INTEREST}

Beckmann and Kääb had received grants from ANR SCD Gene (01 KU 0907), M ${ }^{4}$ Innovative Therapiestrategien für Herzrhythmusstörungen und Drug Safety (01 EX 1021 E) and NGFN Plus of the BMBF (01 GS 0838). Kääb is a Principal Investigator of the Munich Heart Alliance and receives grants from the DZHK (German Centre for Cardiovascular Research). Wilde is a member of the Scientific Advisory board of Transgenomics and Sorin.

\section{ACKNOWLEDGEMENTS}

This work was supported by EuroGentest2 (Unit 2:'Genetic testing as part of health care'), a Coordination Action under FP7 (Grant Agreement Number 261469) and the European Society of Human Genetics.

1 Splawski I, Shen J, Timothy KW et al: Spectrum of mutations in long-QT syndrome genes. KVLQT1, HERG, SCN5A, KCNE1, and KCNE2. Circulation 2000; 102: $1178-1185$.

2 Tester DJ, Will ML, Haglund CM, Ackerman MJ: Compendium of cardiac channel mutations in 541 consecutive unrelated patients referred for long QT syndrome genetic testing. Heart Rhythm 2005; 2: 507-517.

3 Kapplinger JD, Tester DJ, Salisbury BA et al: Spectrum and prevalence of mutations from the first 2500 consecutive unrelated patients referred for the FAMILION Iong QT syndrome genetic test. Heart Rhythm 2009; 6: 1297-1303.

4 Schwartz PJ, Stramba-Badiale M, Crotti L et al: Prevalence of the congenital long-QT syndrome. Circulation 2009; 120: 1761-1767.

5 Kapa S, Tester DJ, Salisbury BA et al: Genetic testing for long-QT syndrome: distinguishing pathogenic mutations from benign variants. Circulation 2009; 120: 1752-1760

6 Napolitano C, Priori SG, Schwartz PJ, Bloise R et al: Genetic testing in the long QT syndrome: development and validation of an efficient approach to genotyping in clinical practice. JAMA 2005; 294: 2975-2980.

7 Priori SG, Schwartz PJ, Napolitano C et al: Risk stratification in the long-QT syndrome. N Engl J Med 2003; 348: 1866-1874.

8 Goldenberg I, Moss AJ, Bradley J et al: Long-QT syndrome after age 40. Circulation 2008; 117: 2192-2201.

9 Hofman N, Wilde AA, Tan HL: Diagnostic criteria for congenital long QT syndrome in the era of molecular genetics: do we need a scoring system? Eur Heart J. 2007; 28: 1399.

10 Ackerman MJ, Priori SG, Willems S et al: HRS/EHRA expert consensus statement on the state of genetic testing for the channelopathies and cardiomyopathies this document was 
developed as a partnership between the Heart Rhythm Society (HRS) and the European Heart Rhythm Association (EHRA). Heart Rhythm 2011; 8: 1308-1339.

11 Wilde AAM, Jongbloed RJE, Doevendans PA et al: Auditory stimuli as a trigger for arrhythmic events differentiate HERG-related (LQTS2) patients from KVLQT1-related patients (LQTS1). J Am Coll Cardiol 1999; 33: 327-332.
12 Schwartz PJ, Priori SG, Spazzolini C et al: Genotype-phenotype correlation in the longQT syndrome: gene-specific triggers for life-threatening arrhythmias. Circulation 2001 103: 89-95.

13 Brink PA, Schwartz PJ: Of founder populations, long QT syndrome, and destiny. Heart Rhythm 2009; 6:11 Suppl S25-S33. 